K. Azukawa and M. Suzuki

Nagoya Math. J.

Vol. 89 (1983), 1-11

\title{
THE BERGMAN METRIC ON A THULLEN DOMAIN
}

\author{
KAZUO AZUKAWA AND MASAAKI SUZUKI
}

\section{§1. Introduction}

In this paper we shall study the holomorphic sectional curvature of the Bergman metric on a domain

$$
D_{p}:=\left\{\left.(z, w) \in C^{2}|| z|<1,| w\right|^{2}<\left(1-|z|^{2}\right)^{p}\right\}
$$

in $C^{2}$, where $0 \leqq p \leqq 1$. (If $p \neq 0$ then

$$
\left.D_{p}=\left\{\left.(z, w) \in C^{2}|| z\right|^{2}+|w|^{2 / p}<1\right\} .\right)
$$

If $0<p<1$ then $D_{p}$ is called a Thullen domain. $\left(D_{0}\right.$ is the unit bidisc and $D_{1}$ the unit ball.)

We shall determine the maximum and the minimum of the curvature at an arbitrary point of $D_{p}$ (Theorem 1), and examine the boundary behavior of the curvature (Corollary of Theorem 2).

We shall have the maximum and the minimum of the curvature on $D_{p}$, which are negative and given by simple rational functions of $p$ (Theorem 3).

\section{§2. Bergman metric on a complete Reinhardt bounded domain in $C^{2}$}

Let $D$ be a bounded domain in $C^{n}$ with the natural coordinate $\left(z^{1}, \cdots, z^{n}\right)$ and $K\left(z^{1}, \cdots, z^{n}\right)$ be the Bergman kernel function of $D$. The Bergman metric on $D$ is defined by

$$
h:=2 \sum_{a, b} h_{a b} d z^{a} \cdot d \bar{z}^{b},
$$

where $h_{a \bar{b}}:=\partial^{2} \log K / \partial z^{a} \partial \bar{z}^{b}$. The Riemann curvature tensor of the metric is given by

$$
R_{a \bar{b} c d}:=\frac{\partial^{2} h_{a \bar{b}}}{\partial z^{c} \partial \bar{z}^{d}}-\sum_{e, f} h^{e \bar{\jmath}} \frac{\partial h_{a \bar{\jmath}}}{\partial z^{c}} \frac{\partial h_{e \bar{b}}}{\partial \bar{z}^{d}},
$$

Received December 3, 1979. 
where $\left(h^{e \vec{f}}\right)$ is the inverse matrix of $\left(h_{a \bar{b}}\right)$ in the sense that $\sum_{b} h_{a \bar{b}} h^{c \bar{b}}=\delta_{a}^{c}$. The holomorphic sectional curvature of the Bergman metric in a direction $u$ at $q \in D$, which is a holomorphic tangent vector at $q$ (i.e. $u \in T_{q}(D)$ ) such that $h(q)(u, \vec{u})=1$, is given by

$$
H(q ; u):=-\sum_{a, b, c, d} R_{a \bar{b} c \bar{d}}(q) u^{a} \bar{u}^{b} u^{c} \bar{u}^{d},
$$

where $u=\sum_{a} u^{a}\left(\partial / \partial z^{a}\right)_{q}$. We use the following notations:

$$
\begin{aligned}
& K_{a}:=\partial K / \partial z^{a}, \quad K_{\bar{a}}:=\partial K / \partial \bar{z}^{a} \quad \text { for } a=1, \cdots, n ; \\
& K_{a_{1} \cdots a_{s} a}:=\partial K_{a_{1} \cdots a_{s}} / \partial z^{a}, \quad K_{a_{1} \cdots a_{s} \bar{a}}:=\partial K_{a_{1} \cdots a_{s}} / \partial \bar{z}^{a} \\
& \quad \text { for } a_{j}=1, \cdots, n, 1, \cdots, \bar{n} \text { and } a=1, \cdots, n .
\end{aligned}
$$

Then the following formulas hold (cf. Kobayashi [4], p. 275):

$$
\begin{aligned}
& h_{a \bar{b}}=\frac{K K_{a \bar{b}}-K_{a} K_{\bar{b}}}{K^{2}} . \\
& R_{a \bar{b} c \bar{d}}=-\left(h_{a \bar{b}} h_{c \bar{d}}+h_{a \bar{d}} h_{c \bar{b}}\right)+\hat{R}_{a \bar{b} c \bar{d}},
\end{aligned}
$$

where

$$
\begin{aligned}
\hat{R}_{a \bar{b} c \bar{d}}:= & \frac{K_{a \bar{b} c \bar{d}}}{K}-\frac{K_{a c} K_{\bar{b} \bar{d}}}{K^{2}} \\
& -\frac{1}{K^{4}} \sum_{e, f} h^{e \bar{f}}\left(K K_{a c \bar{f}}-K_{a c} K_{\bar{f}}\right)\left(K K_{\bar{b} \bar{d} e}-K_{\bar{b} \bar{d}} K_{e}\right) .
\end{aligned}
$$

Suppose $D$ is a complete Reinhardt bounded domain. Since then $K$ is a $C^{\infty}$-function of the variables $\left|z^{j}\right|^{2}(j=1, \cdots, n)$, making use of (2.1), we have

$$
\hat{R}_{a \bar{b} c \bar{d}}=\hat{R}_{c \bar{b} a \bar{d}}, \quad \hat{R}_{a \bar{b} c \bar{d}}=\hat{R}_{a \bar{d} c \bar{b}}, \quad \hat{R}_{a \bar{b} c \bar{d}}=\overline{\hat{R}_{b \bar{a} d \bar{c}}} .
$$

If $n=2$, making use of (2.2), we obtain the following:

Lemma 1. If $D$ is a complete Reinhardt bounded domain in $C^{2}$ then

$$
\begin{aligned}
2- & H\left(q ; u\left(\partial / \partial z^{1}\right)_{q}+v\left(\partial / \partial z^{2}\right)_{q}\right) \\
= & \hat{R}_{1 \overline{1} \overline{1}}(q)|u|^{4}+4 \hat{R}_{1 \overline{1} \overline{2}}(q)|u|^{2}|v|^{2}+\hat{R}_{2 \overline{2} 2 \overline{2}}(q)|v|^{4} \\
& +2 \operatorname{Re}\left(2 \hat{R}_{1 \overline{1} 1 \overline{2}}(q) u^{2} \overline{u v}+\hat{R}_{1 \overline{1} 1 \overline{2}}(q) u^{2} \bar{v}^{2}+2 \hat{R}_{1 \overline{2} 2 \overline{2}}(q) u v \bar{v}^{2}\right)
\end{aligned}
$$

where $q \in D,(u, v) \in C^{2}$ with

$$
h_{1 \overline{1}}(q)|u|^{2}+2 \_\operatorname{Re}\left(h_{1 \overline{2}}(q) u \bar{v}\right)+h_{2 \overline{2}}(q)|v|^{2}=1
$$

and $\hat{R}_{a \bar{b} c \bar{d}}$ is the tensor defined by (2.1). 


\section{§3. Upper and lower curvatures of a bounded domain}

Let $D$ be an arbitrary bounded domain in $C^{n}$. Let $h$ be the Bergman metric on $D$ and $H(q ; u)$ the holomorphic sectional curvature of $h$ in a direction $u$ at $q \in D$. We shall use the following:

Definition. Set

$$
\begin{aligned}
U_{D}(q) & :=\max \left\{H(q ; u) \mid u \in T_{q}(D), h(q)(u, \bar{u})=1\right\}, \\
L_{D}(q) & :=\min \left\{H(q ; u) \mid u \in T_{q}(D), h(q)(u, \bar{u})=1\right\}, \quad q \in D ; \\
u_{D} & :=\sup \left\{U_{D}(q) \mid q \in D\right\}, \\
\ell_{D} & :=\inf \left\{L_{D}(q) \mid q \in D\right\} .
\end{aligned}
$$

We call $U_{D}(q), L_{D}(q), u_{D}$ and $\ell_{D}$ the upper, the lower curvature at $q$, the upper and the lower curvature of $D$ respectively.

The upper and the lower curvatures are biholomorphically invariant quantities on the bounded domains in a fixed $\boldsymbol{C}^{n}$ :

Proposition. Let $f$ be a biholomorphic mapping of $D$ to $\hat{D}$, where $D$ and $\hat{D}$ are bounded domains in $C^{n}$. Then $U_{D}=U_{\hat{D}} \circ f, L_{D}=L_{\hat{D}} \circ f, u_{D}=u_{\hat{D}}$ and $\ell_{D}=\ell_{\hat{D}}$.

Proof. Let $h$ and $\hat{h}$ be the Bergman metrics on $D$ and $\hat{D}$ respectively, and $H_{h}, H_{\hat{h}}$ and $H_{f^{*} \hat{h}}$ the holomorphic sectional curvatures of $h, \hat{h}$ and $f^{*} \hat{h}$ respectively. Then $h=f^{*} \hat{h}$. If $u \in T_{q}(D)(q \in D)$ and $h(q)(u, \bar{u})=1$ then $\hat{h}(f(q))\left(f_{*} u, \overline{f_{*} u}\right)=\left(f^{*} \hat{h}\right)(q)(u, \bar{u})=h(q)(u, \bar{u})=1$. Hence the fact $H_{h}(q ; u)$ $=H_{f^{*} \hat{h}}(q ; u)=H_{\hat{h}}\left(f(q) ; f_{*} u\right)$ implies our assertion.

Q.E.D.

\section{$\S 4$. Upper and lower curvatures at a point of $D_{p}$}

We now return to our domain $D_{p}$ defined in the section 1 . The Bergman kernel function of $D_{p}$ is given by

$$
K(z, w)=c \frac{\left(1-|z|^{2}\right)^{p}-r|w|^{2}}{\left(\left(1-|z|^{2}\right)^{p}-|w|^{2}\right)^{3}\left(1-|z|^{2}\right)^{2-p}}, \quad(z, w) \in D_{p},
$$

where $1 / c\left(=\pi^{2} /(1+p)\right)$ is the volume of $D_{p}$ with respect to the euclidean metric on $C^{2}$ and

$$
r=r(p):=(1-p) /(1+p)
$$

(cf. Ise[2]). The group of all biholomorphic transformations of $D_{p}$ includes the group of the mappings 


$$
\left\{\begin{array}{l}
z^{\prime}=\lambda(z+\alpha) /(1+\bar{\alpha} z), \\
w^{\prime}=\mu\left(1-|\alpha|^{2}\right)^{p / 2}(1+\bar{\alpha} z)^{-p} w,
\end{array}\right.
$$

where $\lambda, \mu, \alpha \in C ;|\lambda|=|\mu|=1,|\alpha|<1$ (cf. Ise[2], p. 517). Now we set

$$
U_{p}:=U_{D_{p}}, \quad L_{p}:=L_{D_{p}}, \quad u_{p}:=u_{D_{p}}, \quad \ell_{p}:=\ell_{D_{p}} .
$$

Lemma 2. If $(z, w) \in D_{p}$ then

$$
\begin{aligned}
& U_{p}(z, w)=U_{p}\left(0,|w|\left(1-|z|^{2}\right)^{-p / 2}\right), \\
& L_{p}(z, w)=L_{p}\left(0,|w|\left(1-|z|^{2}\right)^{-p / 2}\right) .
\end{aligned}
$$

Proof. Let $\left(z_{0}, w_{0}\right) \in D_{p}$. Set

$$
f(z, w):=\left(\left(z-z_{0}\right) /\left(1-\bar{z}_{0} z\right), \mu\left(1-\left|z_{0}\right|^{2}\right)^{p / 2}\left(1-\bar{z}_{0} z\right)^{-p} w\right),
$$

where $\mu:=\left|w_{0}\right| / w_{0}$ if $w_{0} \neq 0$, or $\mu:=1$ if $w_{0}=0$. Then $f$ satisfies the condition (4.3) and maps $\left(z_{0}, w_{0}\right)$ to $\left(0,\left|w_{0}\right|\left(1-\left|z_{0}\right|^{2}\right)^{-p / 2}\right)$. Therefore, Proposition in the previous section implies our assertion.

Q.E.D.

By virtue of Lemma 2, for the purpose of finding the values $U_{p}(z, w)$ and $L_{p}(z, w)$, it is enough to examine $U_{p}$ and $L_{p}$ at $(0, w)$ with $|w|<1$. For the convenience of calculations we introduce a new variable

$$
t=t(w):=\left(1-|w|^{2}\right) /\left(1-r|w|^{2}\right), \quad|w|<1,
$$

where $r=(1-p) /(1+p)$ as (4.2).

Lemma 3. Let $0<p \leqq 1$ and $|w|<1$. If $r$ and $t$ are as (4.2) and (4.5) then

$$
\begin{aligned}
& 2-U_{p}(0, w)=4 \min \left\{A x^{2}+2 B x y+C y^{2} \mid x, y \geqq 0, \alpha x+\beta y=1\right\}, \\
& 2-L_{p}(0, w)=4 \max \left\{A x^{2}+2 B x y+C y^{2} \mid x, y \geqq 0, \alpha x+\beta y=1\right\},
\end{aligned}
$$

where

$$
\left\{\begin{array}{l}
\alpha=3+r t^{2}, \quad \beta=3-r t^{2} ; \\
A=6+4 r t^{2}+(1+r) r t^{3}, \\
B=2\left(9+3 r t^{2}-3(1+r) r t^{3}+2 r^{2} t^{4}\right) /\left(3+r t^{2}\right), \\
C=3\left(6-6 r t^{2}+(1+r) r t^{3}\right) /\left(3-r t^{2}\right) .
\end{array}\right.
$$

Proof. We note $0 \leqq r<1$, because $p>0$. Then $0<t \leqq 1$ and $|w|^{2}$ $=(1-t) /(1-r t)$. It follows that 


$$
\begin{aligned}
& \left\{\begin{array}{l}
h_{1 \overline{1}}(0, w)=\alpha /(1+r) t, \\
h_{2 \overline{2}}(0, w)=\beta(1-r t)^{2} /(1-r)^{2} t^{2}, \\
h_{1 \overline{2}}(0, w)=0 ;
\end{array}\right. \\
& \left\{\begin{array}{l}
\hat{R}_{1 \overline{1} 1 \overline{1}}(0, w)=4 A /(1+r)^{2} t^{2}, \\
\hat{R}_{1 \overline{1} \overline{2}}(0, w)=2(1-r t)^{2} B /(1+r)(1-r)^{2} t^{3}, \\
\hat{R}_{2 \overline{2} \overline{2} \overline{2}}(0, w)=4(1-r t)^{4} C /(1-r)^{4} t^{4}, \\
\hat{R}_{1 \overline{1} \overline{1} \overline{2}}(0, w)=0, \\
\hat{R}_{1 \overline{2} \overline{1} \overline{2}}(0, w)=0, \\
\hat{R}_{1 \overline{2} 2 \overline{2} \overline{2}}(0, w)=0 .
\end{array}\right.
\end{aligned}
$$

Setting $x:=|u|^{2} /(1+r) t, y:=|v|^{2}(1-r t)^{2} /(1-r)^{2} t^{2}$, we obtain the desired formulas by Lemma 1 .

Q.E.D.

Now our key theorem is the following:

THEOREM 1. Let $0 \leqq p \leqq 1$ and $|w|<1$. If $r$ and $t$ are as (4.2) and (4.5) then

$$
\begin{aligned}
& U_{p}(0, w)=2-4 F /\left(3+r t^{2}\right)^{2} E, \\
& L_{p}(0, w)=2-4 \max \left\{3\left(6-6 r t^{2}+(1+r) r t^{3}\right) /\left(3-r t^{2}\right)^{3},\right. \\
& \left.\left(6+4 r t^{2}+(1+r) r t^{3}\right) /\left(3+r t^{2}\right)^{2}\right\},
\end{aligned}
$$

where

$$
\begin{aligned}
E= & 162(1+r)-180 r t-81(1+r) r t^{2}+48 r^{2} t^{3}+24(1+r) r^{2} t^{4} \\
& -12 r^{3} t^{5}-(1+r) r^{3} t^{6}>0, \\
F= & 972(1+r)-1080 r t+162(1+r) r t^{2}-27\left(3(1+r)^{2}+16 r\right) r t^{3} \\
& +72(1+r) r^{2} t^{4}+18\left(3(1+r)^{2}-4 r\right) r^{2} t^{5}-54(1+r) r^{3} t^{6} \\
& +\left(3(1+r)^{2}+16 r\right) r^{3} t^{7} .
\end{aligned}
$$

To prove Theorem 1, we prepare the following:

Lemma 4. Let $\alpha, \beta, A, B$ and $C$ be real numbers such that $\alpha, \beta, C \alpha-B \beta$ and $A \beta-B \alpha$ are all positive. Set $f(x, y):=A x^{2}+2 B x y+C y^{2}, g(x, y):=$ $\alpha x+\beta y$. Then we have

$$
\begin{aligned}
& \max \{f(x, y) \mid x, y \geqq 0, g(x, y)=1\}=\max \left\{A / \alpha^{2}, C / \beta^{2}\right\}, \\
& \min \{f(x, y) \mid x, y \geqq 0, g(x, y)=1\}=\frac{A C-B^{2}}{A \beta^{2}-2 B \beta \alpha+C \alpha^{2}} .
\end{aligned}
$$

Proof. Using $A / \alpha^{2}, B / \beta^{2} \geqq\left(A C-B^{2}\right) /\left(A \beta^{2}-2 B \beta \alpha+C \alpha^{2}\right)$, we obtain our assertion by the Lagrange's method.

Q.E.D. 
Proof of Theorem 1. Suppose $0<p \leqq 1$. Let $\alpha, \beta, A, B$ and $C$ be as (4.6). It follows that

$$
\begin{aligned}
& C \alpha-B \beta=r t^{3} E_{1} /\left(3-r t^{2}\right)\left(3+r t^{2}\right), \quad A \beta-B \alpha=r t^{3} E_{2}, \\
& \mathrm{~A} \beta^{2}-2 B \beta \alpha+C \alpha^{2}=\beta(A \beta-B \alpha)+\alpha(C \alpha-B \beta)=r t^{3} E /\left(3-r t^{2}\right), \\
& E=\left(3-r t^{2}\right)^{2} E_{2}+E_{1}, \\
& A C-B^{2}=r t^{3} F /\left(3-r t^{2}\right)\left(3+r t^{2}\right)^{2},
\end{aligned}
$$

where

$$
\left\{\begin{aligned}
& E_{1}:=9 E_{11}+E_{12} r^{2} t^{4}, \quad E_{11}:=9(1+r)-12 r t-9(1+r) r t^{2}, \\
& E_{12}:=9(1+r)-4 r t \\
& E_{2}:=9(1+r)-8 r t-(1+r) r t^{2}
\end{aligned}\right.
$$

If $0<p<1$ then $0<r<1$ and $E_{11}, E_{12}, E_{2}>0(0<t \leqq 1)$. Moreover $C \alpha-B \beta>0, A \beta-B \alpha>0$. Applying Lemma 4 to the above values, we obtain the desired formulas in the case $0<p<1$.

If $p=1$ then $r=0$. In this case we can prove our assertion directly from Lemma 3.

Suppose $p=0$. Then $t=1$ identically. But we know that $U_{0}(0, w)$ $=-1 / 2, L_{0}(0, w)=-1$ (cf. Kobayashi [5], p. 40). Hence our assertion is valid also for $p=0$.

Q.E.D.

\section{§5. Upper and lower curvatures of $D_{p}$}

From Theorem 1 we induce some consequences.

Theorem 2. Let $0<p<1$. Then:

(i ) $\lim _{|w| \rightarrow 1} L_{p}(0, w)=\lim _{|w| \rightarrow 1} U_{p}(0, w)=-2 / 3$.

(ii) $L_{p}(0, w)$ is strictly increasing with respect to $|w|$.

(iii) $U_{p}(0, w)$ is strictly decreasing with respect to $|w|$.

Proof. (i): Obvious by Theorem 1 .

(ii): If $0<r<1$ and $0<t \leqq 1$ then

$$
\begin{aligned}
& \frac{\partial}{\partial t}\left(\frac{6-6 r t^{2}+(1+r) r t^{3}}{\left(3-r t^{2}\right)^{3}}\right)=\frac{3 r t^{2}\left(3(1+r)-8 r t+(1+r) r t^{2}\right)}{\left(3-r t^{2}\right)^{4}}>0, \\
& \frac{\partial}{\partial t}\left(\frac{6+4 r t^{2}+(1+r) r t^{3}}{\left(3+r t^{2}\right)^{2}}\right)=\frac{r t^{2}\left(9(1+r)-8 r t-(1+r) r t^{2}\right)}{\left(3+r t^{2}\right)^{3}}>0 .
\end{aligned}
$$

It follows that $L_{p}(0, w)$ is strictly decreasing with respect to $t$.

(iii): If $0<r<1$ and $0<t \leqq 1$ then 


$$
\begin{aligned}
\frac{\partial}{\partial t}\left(F /\left(3+r t^{2}\right)^{2} E\right) & =\left(\left(\frac{\partial F}{\partial t} E-F \frac{\partial E}{\partial t}\right)\left(3+r t^{2}\right)-4 r t E F\right) /\left(3+r t^{2}\right)^{3} E^{2} \\
& =r t^{2} M /\left(3+r t^{2}\right)^{3} E^{2}
\end{aligned}
$$

where

$$
\left\{\begin{aligned}
M:= & 9^{2} M_{1}+9 r^{4} t^{7} M_{2}+3 r^{6} t^{11} M_{3}, \\
M_{1}:= & -2 \cdot 9^{3}\left(1+3 r+3 r^{2}+r^{3}\right)+56 \cdot 9^{3}\left(r+2 r^{2}+r^{3}\right) t \\
& +5 \cdot 9\left(45 r+23 r^{2}+23 r^{3}+45 r^{4}\right) t^{2}-48\left(123 r^{2}+206 r^{3}+123 r^{4}\right) t^{3} \\
& -3\left(141 r^{2}-1385 r^{3}-1385 r^{4}+141 r^{5}\right) t^{5}+48\left(13 r^{3}-6 r^{4}+13 r^{5}\right) t^{5} \\
& +6\left(31 r^{3}+109 r^{4}+109 r^{5}+31 r^{6}\right) t^{6}-32\left(9 r^{4}+26 r^{5}+5 r^{6}\right) t^{7} \\
& \\
M_{2}:= & -32 \cdot 9 \cdot 4 r^{2}-16 \cdot 9\left(3-r-r^{2}+3 r^{3}\right) t+8\left(75+86 r^{2}+75 r^{3}\right) t^{2} \\
& +\left(21 r-241 r^{2}-241 r^{3}+21 r^{4}\right) t^{3}-r^{2} t^{4}, \\
M_{3}:= & \left(-45+32 r-48 r^{2}\right)+\left(3+25 r+25 r^{2}+3 r^{3}\right) t .
\end{aligned}\right.
$$

But it can be proved that $M_{1}, M_{2}, M_{3}<0$ for $0<r<1$ and $0<t \leqq 1$. As the authors' proof is tedious, we leave it in Appendices (Proposition A3 and Proposition A4). Admitting the above facts, we conclude our assertion by a similar proof to (ii).

Q.E.D.

Instead of (iii) the following is more easily proved:

(iii') $U_{p}(0, w)>-2 / 3$ for $|w|<1$;

which we shall use in the following:

Corollary. Let $0<p<1$. Let $H_{p}(z, w ; u)$ be the holomorphic sectional curvature of the Bergman metric on $D_{p}$ in a direction $u$ at $(z, w) \in$ $D_{p}$. Let $(\zeta, \omega) \in \partial D_{p}$. Then:

(i) If $\omega \neq 0$ then $\lim _{(z, w) \rightarrow(\zeta, \omega)} H_{p}(z, w ; u)=-2 / 3$ uniformly in the directions $u$.

(ii) If $\omega=0$ then there does not exist the uniform limit of $H_{p}(z, w ; u)$ as $(z, w) \rightarrow(\zeta, 0)$.

Proof. By Lemma 2 the image of the mapping $u \mapsto H_{p}(z, w ; u)$ is the closed interval $\left[L_{p}\left(0,|w|\left(1-|z|^{2}\right)^{-p / 2}\right), U_{p}\left(0,|w|\left(1-|z|^{2}\right)^{-p / 2}\right)\right]$.

(i): If $\omega \neq 0$ then $|w|\left(1-|z|^{2}\right)^{-p / 2} \rightarrow 0$ as $(z, w) \rightarrow(\zeta, \omega)$, hence $\operatorname{Im} H_{p}(z, w ; \cdot) \rightarrow\{-2 / 3\}$ as $(z, w) \rightarrow(\zeta, \omega)$ by (i), (ii) in Theorem 2 and (iii').

(ii): If $\omega=0$ and a complex sequence $\left(z_{j}\right)$ satisfies $\left|z_{j}\right|<1, z_{j} \rightarrow \zeta$ then $\operatorname{Im} H_{p}\left(z_{j}, 0 ; \cdot\right)=\left[L_{p}(0,0), U_{p}(0,0)\right] \supseteq\{-2 / 3\}$ by (i), (ii) in Theorem 2 and (iii').

Q.E.D.

Remark. If $D \subset C^{n}$ is a strongly pseudoconvex bounded domain with 
$C^{\infty}$ boundary and if $\hat{q} \in \partial D$ then $\lim _{q \rightarrow \hat{q}} H(q, u)=-2 /(n+1)$ uniformly in the directions $u$ (cf. Theorem 1 in Klembeck [3]). In our domain $D_{p}$, suppose $1 / p$ be a positive integer. Then $D_{p}$ is with $C^{\infty}$ boundary and is strongly pseudoconvex at $(\zeta, \omega) \in \partial D_{p}$ if and only if $\omega \neq 0$. Corollary gives a counter example to the question whether the above theorem is valid under the assumption that $D$ is pseudoconvex instead of strongly pseudoconvex.

As an immediate consequence of Theorem 2, we obtain:

Theorem 3. Let $0 \leqq p \leqq 1$. Then:

(i) $\ell_{p}=L_{p}(0,0)=-\left(1+4 p+p^{2}\right) /(1+2 p)^{2}$.

(ii) $u_{p}=U_{p}(0,0)=-2\left(2+11 p+15 p^{2}+8 p^{3}\right) /(2+p)(1+3 p)(4+5 p)$.

Instead of (ii) the following is more easily proved:

(ii') $u_{p}=\max \left\{U_{p}(0, w)|| w \mid<1\right\}<0$.

According to Proposition in the section 3, we obtain:

CoRollary 1. If $0 \leqq p_{1}<p_{2} \leqq 1$, then $\ell_{p_{1}}<\ell_{p_{2}}$, hence $D_{p_{1}}$ is not biholomorphically equivalent to $D_{p_{2}}$.

From (ii') we have the following:

CoRollary 2. Let $0 \leqq p \leqq 1$. The holomorphic sectional curvature of the Bergman metric on $D_{p}$ is strictly negative.

\section{Appendices}

\section{A1. Fourier's theorem concerning to the zeros of a polynomial}

Set $\operatorname{sgn} c:=c /|c|, c \in \boldsymbol{R}-\{0\}$. Let $q$ be the number of the non-zero terms in a real finite sequence $\left(c_{j}\right)_{j=0}^{p}$. We define the number of changes of sign in $\left(c_{j}\right)$ as follows:

$$
V\left(c_{0}, \cdots, c_{p}\right):=\left\{\begin{array}{cl}
\sum_{j=1}^{q-1}\left(1-\operatorname{sgn} c_{n_{j-1}} c_{n_{j}}\right) / 2, & q \geqq 2, \\
0, & q=0 \text { or } 1,
\end{array}\right.
$$

where if $q \geqq 1,\left(c_{n_{j}}\right)_{j=0}^{q-1}$ is the subsequence deleted the terms $c_{j}$ with $c_{j}=0$ (i.e. $n_{0}=\min \left\{k \mid c_{k} \neq 0\right\}, n_{j}=\min \left\{k>n_{j-1} \mid c_{k} \neq 0\right\}(1 \leqq j \leqq q-1)$ ).

Let $f \in \boldsymbol{R}[t]-\{0\}, c \in \boldsymbol{R}$ and $I \subset \boldsymbol{R}$ be an interval. We denote

$$
\begin{aligned}
& V(c):=V_{f}(c):=V\left(f(c), f^{(1)}(c), \cdots, f^{(n)}(c)\right), n:=\operatorname{deg} f ; \\
& N I:=N_{f} I:=\sum_{t \in I}(\text { the order of zero to } f \text { at } t) .
\end{aligned}
$$


The following theorem is well known:

FourieR's Theorem ([1]). Let $f \in \boldsymbol{R}[t]-\{0\}$ and $a, b \in \boldsymbol{R}$ with $a<b$. Then there is a non-negative integer $\nu$ such that

$$
N(a, b]=V(a)-V(b)-2 \nu .
$$

As an immediate consequence of Fourier's Theorem we have:

Proposition A1. Let $f, a$ and $b$ be as in Fourier's Theorem. Then:

(i) If $V(a)=V(b)$, then $f$ has no zero in $(a, b]$.

(ii) If $V(a)=V(b)+1$, then $f$ has only one simple zero in $(a, b]$.

We shall use Proposition A1 in the following section.

\section{A2. Negativity of $M_{j}$ in the proof of Theorem 3}

In this section we shall show that the functions $M_{j}$ of the variables $r$ and $t$ defined by (5.1) are negative for $(r, t) \in(0,1]^{2}$. First we can write

$$
\frac{\partial M_{1}}{\partial t}=6 r N_{1}+4 r^{4} t^{5} N_{2}
$$

where

$$
\left\{\begin{aligned}
N_{1}:= & 756\left(1+2 r+r^{2}\right)+15\left(45+23 r+23 r^{2}+45 r^{3}\right) t \\
& -24\left(123 r+206 r^{2}+123 r^{3}\right) t^{2}-2\left(141 r-1385 r^{2}-1385 r^{3}+141 r^{4}\right) t^{3} \\
& +40\left(13 r^{2}-6 r^{3}+13 r^{4}\right) t^{4}+186 r^{2} t^{5} \\
N_{2}:= & 9\left(109+109 r+31 r^{2}\right)-56\left(9+26 r+5 r^{2}\right) t .
\end{aligned}\right.
$$

Proposition A2. $N_{1}(r, t)>0$ for $(r, t) \in(0,1]^{2}$.

Proof. Set $f_{r}(t):=N_{1}(r, t),(r, t) \in(0,1]^{2}$. We shall apply Proposition A1 to $f_{r}$ and the interval $(0,1]$. It follows that

$$
\begin{aligned}
& f_{r}^{(j)}(0)=j !\left(\text { the coefficient of } t^{j} \text { in } f_{r}\right) \\
& f_{r}(1)=1431-1377 r-367 r^{2}+253 r^{3}+238 r^{4}, \\
& f_{r}^{(1)}(1)=675-6405 r+1777 r^{2}+2121 r^{3}+1234 r^{4} \\
& f_{r}^{(2)}(1)=12 r\left(-633+1391 r+653 r^{2}+379 r^{3}\right), \\
& f_{r}^{(3)}(1)=12 r\left(-141+3355 r+905 r^{2}+899 r^{3}\right), \\
& f_{r}^{(4)}(1)=240 r^{2}\left(145-24 r+52 r^{2}\right), \\
& f_{r}^{(5)}(1)=240.93 r^{2} .
\end{aligned}
$$

Applying Proposition A1 to the polynomials $f_{r}^{(j)}(0), f_{r}^{(j)}(1)$ of variable $r$ and 
the interval $(0,1]$, we can see that $f_{r}(0), f_{r}^{(1)}(0), f_{r}^{(2)}(0), f_{r}^{(4)}(0), f_{r}^{(5)}(0), f_{r}(1)$, $f_{r}^{(4)}(1)$ and $f_{r}^{(5)}(1)$ have no zero in $(0,1]$, while each of $f_{r}^{(3)}(0), f_{r}^{(1)}(1), f_{r}^{(2)}(1)$ and $f_{r}^{(3)}(1)$ has only one simple zero in $(0,1]$, say $r_{1}, r_{2}, r_{3}$ and $r_{4}$ respectively. Moreover we have

$$
0<r_{4}<\frac{1}{20}<r_{1}<\frac{1}{10}<r_{2}<\frac{1}{5}<r_{3}<1
$$

and the following tables of signs:

\begin{tabular}{l|l}
\hline \multicolumn{1}{c|}{} & $0 \quad r_{1} 1$ \\
\hline$f_{r}(0)$ & $:+:+:$ \\
$f_{r}^{(1)}(0)$ & $:+:+:$ \\
$f_{r}^{(2)}(0)$ & $0-:-:$ \\
$f_{r}^{(3)}(0)$ & $0-0+:$ \\
$f_{r}^{(4)}(0)$ & $0+:+:$ \\
$f_{r}^{(5)}(0)$ & $0+:+:$ \\
\hline
\end{tabular}

Table 1.

\begin{tabular}{|c|c|}
\hline$r$ & $\begin{array}{lllll}0 & r_{4} & r_{2} & r_{3} & 1\end{array}$ \\
\hline$f_{r}(1)$ & $:+:+:+:+:$ \\
\hline$f_{r}^{(1)}(1)$ & $:+:+0-:-:$ \\
\hline$f_{r}^{(2)}(1)$ & $0-:-:-0+:$ \\
\hline$f_{r}^{(3)}(1)$ & $0-0+:+:+:$ \\
\hline$f_{r}^{(4)}(1)$ & $0+:+:+:+:$ \\
\hline$f_{r}^{(5)}(1)$ & $0+:+:+:+:$ \\
\hline
\end{tabular}

Table 2.

It follows from the tables that $V_{f_{r}}(0)=V_{f_{r}}(1)=2, r \in(0,1]$. Therefore $f_{r}$ has no zero in $(0,1]$ for any $r \in(0,1]$.

Q.E.D.

Proposition A3. $M_{1}<0$ for $(r, t) \in(0,1]^{2}$.

Proof. It is easily seen that

$$
N_{2} \geqq N_{2}(r, 1) \geqq N_{2}(1,1)=1 .
$$

Proposition A2 and (A2.1) show that $M_{1}(r, t) \leqq M_{1}(r, 1),(r, t) \in(0,1]^{2}$. But we have

$$
M_{1}(r, 1)=-2 \cdot 9^{3}+3 \cdot 9^{3} r-66 \cdot 9 r^{2}-10 \cdot 9^{2} r^{3}+354 r^{4}+23 r^{5}+26 r^{6} ;
$$

therefore using Proposition A1, we obtain $M_{1}(r, 1)<0, r \in(0,1]$. Q.E.D.

Finally we consider $M_{2}$ and $M_{3}$. Set $g_{r}(t):=M_{2}(r, t),(r, t) \in(0,1]^{2}$. Then $V_{g_{r}}(0)=V_{g_{r}}(1), r \in(0,1]$. On the other hand, $M_{3} \leqq M_{3}(r, 1) \leqq M_{3}(1,1)$ $=-5$. Therefore we have proved the following:

Proposition A4. $M_{2}, M_{3}<0$ for $(r, t) \in(0,1]^{2}$. 


\section{REFERENCES}

[1] Fourier, J., Analyse des équations déterminées, Ostwald's Klassiker, No. 127, 1831.

[2] Ise, M., On Thullen domains and Hirzeburch manifolds I, J. Math. Soc. Japan, 26 (1974), 508-522.

[ 3 ] Klembeck, P. F., Kähler metrics of negative curvature, the Bergman metric near the boundary, and the Kobayashi metric on smooth bounded strictly pseudoconvex sets, Indiana Univ. Math. J., 27 (1978), 275-282.

[4] Kobayashi, S., Geometry of bounded domains, Trans. Amer. Math. Soc., 92 (1959), 267-290.

[5] - Hyperbolic Manifolds and Holomorphic Mappings, Marcel Dekker, New York, 1970.

Lepartment of Mathematics

Toyama University

Gofuku, Toyama, Japan 